\title{
Multifrequency Analysis of a UV Ceti Flare on 1991 December 31
}

\author{
A.V. Stepanov ${ }^{1}$, E. Fürst ${ }^{2}$, A. Krüger ${ }^{3}$, J. Hildebrandt ${ }^{3}$, H. Barwig ${ }^{4}$, \\ J.H.M.M. Schmitt ${ }^{5}$ \\ 1 Crimean Astrophysical Observatory, RT-22 Katzively, 334247 Crimea, Ukraine \\ 2 MPI für Radioastronomie, Auf dem Hügel 69, D-53121 Bonn, Germany \\ 3 Astrophysikalisches Institut, An der Sternwarte 16, D-14482 Potsdam, Germany \\ 4 Institut für Astronomie und Astrophysik der Universität München, Scheinerstr. 1, \\ D-81679 München, Germany \\ 5 MPI für Extraterrestrische Physik, Giessenbachstrasse, D-85748 Garching, Germany
}

\begin{abstract}
An analysis of optical, radio and X-ray observations of the flare of December 31, 1991 on UV Ceti is presented. Radio spikes at $4750 \mathrm{GHz}$ are interpreted in terms of both ECM and plasma emission. Resulting parameters of the flare plasma are discussed.
\end{abstract}

\section{Observations}

Optical data of UV Cet were obtained in $U B V R I$ with the $0.8 \mathrm{~m}$ telescope of the Wendelstein Observatory. Two flares were recognized, near 17:00 and 18:25 UT. The second one was a strong white light flare visible in almost all colors. Radio observations were carried out with the Effelsberg $100 \mathrm{~m}$ radio telescope at $4750 \mathrm{MHz}$ with a total bandwidth of $50 \mathrm{MHz}$ splitted into 32 channels. The time constant was $0.125 \mathrm{~s}$. The burst started about $5 \mathrm{~min}$ after the second optical flare and showed $\gtrsim 75 \% \mathrm{LH}$ circular polarization with a peak flux density of $250 \mathrm{mJy}$. The polarized radio data are integrated to $0.9 \mathrm{~s}$ for various bands each about $3.1 \mathrm{MHz}$ wide and display several larger spikes with duration $\lesssim 0.125 \mathrm{~s}$. Soft $\mathrm{X}$-ray emission $(0.1-2.5 \mathrm{keV})$ was observed with ROSAT PSPC before and after the optical and radio flares only, because UV Cet was unfortunately occulted by the Earth. Nevertheless, the data associate suggestively the decay phase of the $\mathrm{X}$-ray flare with the optical and radio flare observations.

\section{Discussion}

The brightness temperature of the radio spikes was estimated to be $3 \cdot 10^{12} \mathrm{~K}$ indicating coherent radiation. Usually two coherent radiation mechanisms are considered for the spikes: Electron Cyclotron Maser (ECM) and plasma emission. Two problems arise if we assume ECM emission. The first one is that the electron gyrofrequency to plasma frequency ratio should be $\nu_{B} / \nu_{\mathrm{p}}>1$, but the corresponding Alfvén velocity $\left(v_{\mathrm{A}} \approx 10^{4} \mathrm{~km} / \mathrm{s}\right)$ seems to be too large for the stellar corona. The second problem is the escape of the ECM emission from the 
corona, because the emission should be absorbed at the gyroresonance layers $\nu=s \nu_{B}(s=2,3)$. To resolve this problem Robinson (1984) suggested an escape window for the o-mode at $\nu=2 \nu_{B}$ near the direction perpendicular to the magnetic field. However, our calculations of optical depths for gyroabsorption show that there are no "perpendicular" windows for the o-mode at $s=2,3$. Instead, quite large "parallel" windows for both the $\mathrm{x}$ - and o-mode exist, enabling the escape of ECM emission if some wave isotropization mechanism works. Supposing ECM emission, one can derive the magnetic field $B \approx 850 \mathrm{G}$ and the plasma density $n \lesssim 7 \cdot 10^{10} \mathrm{~cm}^{-3}$ in the source region.

It is not excluded that highly polarized spikes are due to o-mode radiation from plasma emission at the fundamental $\nu \approx \nu_{\mathrm{p}}$. Thus, the radio observation corresponds to $n \approx 3 \cdot 10^{11} \mathrm{~cm}^{-3}$, and from the condition for the $\mathrm{x}$-mode cut-off, we estimate $B \gtrsim 200 \mathrm{G}$. Genkin et al. (1990) have shown that emission at $\nu \approx \nu_{\mathrm{p}}$ can be very irregular due to scattering of plasma waves on density irregularities induced by plasma waves themselves and thermodiffusion. The correlation time $t_{\mathrm{c}}=2 v /\left(\nu_{\mathrm{e} i} v_{\mathrm{g}}\right)$ (for $t>t_{\mathrm{c}}$ no spikes exist!) depends on the energetic electron velocity $v$, the group velocity of plasma waves $v_{\mathrm{g}}$ and the electron-ion collisional frequency $\nu_{\mathrm{ei}}$ and is compared with the spike duration observed ( $\left.t_{\mathrm{i}} \lesssim 0.13 \mathrm{~s}\right)$.

From the multifrequency observations of the UV Cet flare on 1991 December 31 we find the following parameters for the radio source: $B \approx 200-300 \mathrm{G}, n \approx$ $10^{11} \mathrm{~cm}^{-3}, T \approx 10^{7} \mathrm{~K}$, and the source size $D \approx 10^{4} \mathrm{~km}$, which do not contradict known diagnostics of flare plasmas on red dwarfs. The temperature of the "cool" plasma deduced from optical data is $T \approx 1.6 \cdot 10^{4} \mathrm{~K}$ and the linear scale $L \approx$ $2 \cdot 10^{3} \mathrm{~km}$. The time delay of about $5 \mathrm{~min}$ between the optical and radio flare can be understood either in terms of magnetic trapping and storage of accelerated particles or in terms of evaporated plasma filling up the flare loop. The flare scenario can be described using both the Impulsive Explosion Model (De Jager et al. 1989) and the Advanced Circuit Model (Zaitsev \& Stepanov 1992). A more detailed discussion will be published elsewhere (Stepanov et al. 1995).

\section{References}

De Jager C., Heise J., van Genderen et al., 1989, A\&A 211, 157

Genkin L.G., Erukhimov L.M., Levin B.N., 1990, Solar Phys. 128, 423

Robinson P.A., 1984, ApJ 341, L99

Stepanov A.V., Fürst E., Krüger A., Hildebrandt J., Barwig H., Schmitt J.H.M.M., 1995, A\&A (submitted)

Zaitsev V.V., Stepanov A.V., 1992, Solar Phys. 139, 343 